\title{
Analysis of Displacement Errors in High-Resolution Image Reconstruction With Multisensors
}

\author{
Michael K. Ng and N. K. Bose, Fellow, IEEE
}

Invited Paper

\begin{abstract}
An image-acquisition system composed of an array of sensors, where each sensor has a subarray of sensing elements of suitable size, has recently been popular for increasing the spatial resolution with high signal-to-noise ratio beyond the performance bound of technologies that constrain the manufacture of imaging devices. Small perturbations around the ideal subpixel locations of the sensing elements (responsible for capturing the sequence of undersampled degraded frames), because of imperfections in fabrication, limit the performance of the signal-processing algorithms for processing and integrating the acquired images for the desired enhanced resolution and quality. The contributions of this paper include an analysis of the displacement errors on the convergence rate of the iterative approach for solving the transform based preconditioned system of equations. Subsequently, it is established that the use of the MAP, $L_{2}$ norm or $H_{1}$ norm regularization functional leads to a proof of linear convergence of the conjugate gradient method in terms of the displacement errors caused by the imperfect subpixel locations. Results of simulation support the analytical results.
\end{abstract}

Index Terms-Displacement-error analysis, high resolutin, image reconstruction, multisensors.

\section{INTRODUCTION}

A VERY fertile arena for applications of some of the developed theory of multidimensional systems has been spatio-temporal processing following image acquisition by, say a single camera, mutiple cameras or an array of sensors. Due to hardware cost, size, and fabrication complexity limitations, imaging systems like CCD detector arrays often provide only multiple low-resolution degraded images. However, a high-resolution image is indispensable in applications including health diagnosis and monitoring, military surveillance, and terrain mapping by remote sensing. Other intriguing possibilities include substituting expensive high resolution instruments like scanning electron microscopes by their cruder, cheaper counterparts and then applying technical methods for increasing the

Manuscript received March 7, 2001; revised January 6, 2002. This work was supported in part by Research Grants Council, Hong Kong under Grant HKU 7147/99P, Grant 7132/00P, and Grant HKU CRCG 10203501, and in part by Army Research Office under Grant DAAD 19-00-1-0539. This paper was recommended by Co-Guest Editor M. N. S. Swamy.

M. K. Ng is with the Department of Mathematics, The University of Hong Kong, Hong Kong (e-mail: mng@maths.hku.hk).

N. K. Bose is with the Department of Electrical Engineering, The Spatial and Temporal Signal Processing Center, The Pennsylvania State University, University Park, PA 16802 USA (e-mail: nkb@stspbkn.ee.psu.edu).

Publisher Item Identifier S 1057-7122(02)05595-2. resolution to that derivable with much more costly equipment. Resolution improvement by applying tools from digital signal processing technique has, therefore, been a topic of very great interest [3], [5], [9], [11], [16], [19], [20], [6]. The attainment of image superresolution (for simplicity, the difference in the terminologies of superresolution and high-resolution or interpolation is ignored, because in both cases the low-resolution images acquired for processing in this paper are assumed to be from sensor arrays which are shifted from each other by subpixel displacements) was based on the feasibility of reconstruction of 2-D bandlimited signals from nonuniform samples [10] arising from frames generated by microscanning i.e., subpixel shifts between successive frames, each of which provides an unique snapshot of a stationary scene.

In [9], Kim, Bose, and Valenzuela proposed a weighted recursive least squares algorithm, based on sequential estimation theory in the frequency-wavenumber domain, to achieve simultaneous improvement in signal-to-noise ratio and resolution from available registered (for horizontal and vertical displacements, sufficient for LANDSAT type imaging) sequence of low-resolution noisy frames. In [11], Kim and Su also incorporated explicitly the deblurring computation into the high-resolution image reconstruction process since separate deblurring of input frames would introduce the undesirable phase and high wavenumber distortions in the DFT of those frames. A DCT-based approach in the spatial domain with regularization, but without the recursive updating feature, was recently considered in [17] and an optimization theory based approach with regularization was given in [7]. Proper choice of the regularization tuning parameter is crucial to achieving robustness in the presence of noise. The $L$-curve based procedure, recently applied to image processing, avoids trial-and-error in the selection of an optimal tuning parameter [2]. Stark and Oskoui [19] and Tekalp, Ozkan and Sezan [20] formulated a projection onto convex sets algorithm to compute an estimate from low-resolution images obtained by either scanning or rotating an image with respect to the CCD image acquisition sensor array or mounting the image on a moving platform [7].

Multiple undersampled images of a scene are often obtained by using multiple identical image sensors which are shifted relative to each other by subpixel displacements [12], [8]. The resulting high-resolution image reconstruction problem using a set of currently-available image sensors is interesting because 
it is closely related to the design of high-definition television (HDTV) and very high-definition (VHD) image sensors. CCD image sensor arrays, where each sensor consists of a rectangular subarray of sensing elements, produce discrete images whose sampling rate and resolution are determined by the physical size of the sensing elements. If multiple CCD image sensor arrays are shifted relative to each other by exact subpixel values, the reconstruction of high-resolution images can be modeled by

$$
\bar{g}=H f \quad \text { and } \quad g=\bar{g}+\eta
$$

where $f$ is the desired high-resolution image, $H$ is the blur operator, $\bar{g}$ is the output high-resolution image formed from low-resolution frames and $\eta$ is the additive Gaussian noise. However, as perfect subpixel displacements are practically impossible to realize, blur operators in multisensor high-resolution image reconstruction are space-variant.

Since the system described in (1) is ill-conditioned, solution for $f$ is constructed by applying the maximum a posteriori (MAP) regularization technique that involves a functional $R(f)$, which measures the regularity of $f$, and a regularization parameter $\alpha$ that controls the degree of regularity of the solution to the minimization problem

$$
\min _{f}\left\{\|H f-g\|_{2}^{2}+\alpha R(f)\right\} .
$$

The boundary values of $g$ are not completely determined by the original image $f$ inside the scene, because of the blurring process. They are also affected by the values of $f$ outside the scene. Therefore, when solving for $f$ from (1), one needs some assumptions on the values of $f$ outside ther scene, referred to as boundary conditions. In [3], Bose and Boo imposed zero boundary condition outside the scene, i.e., a dark background outside the scene was assumed, which can produce a ringing effect at the boundary of the reconstructed image. The problem is aggravated if the images are acquired from a large sensor array since the number of pixel values of the image affected by the sensor array increases (see [4]). In [4], [14], [15], the Neumann boundary condition was imposed i.e., the scene immediately outside is a reflection of the original scene at the boundary. $\mathrm{Ng}$ and Yip [15] recently showed that the model with the Neumann boundary condition gives better reconstructed high-resolution image than obtainable with the zero boundary condition.

The MAP regularization [3], [15], the $L_{2}$ norm regularization functional $\left(R(f)=\|f\|_{2}^{2}\right)$ and $H_{1}$ norm regularization functional $\left(R(f)=\|\mathcal{L} f\|_{2}^{2}\right)$ [14] have been considered and used in high-resolution image reconstruction. Here, $\mathcal{L}$ is the first order differential operator. Moreover, transform based preconditioners were applied to solve the structured linear system of equations arising from high-resolution image reconstruction models. Numerical results have shown that these transform based preconditioners are effective.

In practice, there can be small perturbations around these ideal subpixel locations due to imperfections of the fabrication imaging system. The main aim of this paper is to give a detailed discussion and analysis of these displacement errors in the convergence rate of the iterative method when applied to solve the transform based preconditioned system. We prove that when the MAP, $L_{2}$ norm or $H_{1}$ norm regularization functional is used, the convergence of the conjugate gradient method depends linearly on these displacement errors arising from imperfect subpixel locations. We remark that this analysis has not been discussed in the previous papers.

The outline of the paper is as follows. In Section II, we give a mathematical formulation of the problem. Analysis of displacement errors in the convergence analysis of the iterative method is given in Section III. In Section IV, numerical results are presented to demonstrate the theoretical results shown in Section III.

\section{The MATHematical Model}

In this paper, a specific high-resolution image reconstruction problem, based on a model for a prefabricated multisensor image acquisition system, recently proposed and studied by Bose and Boo [3] is considered. More general high-resolution (or super-resolution) image reconstruction problems can be found in [8], [18], [20].

We begin with a brief introduction to the mathematical model considered by Bose and Boo. Consider a sensor array with $L_{1} \times$ $L_{2}$ sensors in which each sensor has $N_{1} \times N_{2}$ sensing elements (pixels) and the size of each sensing element is $T_{1} \times T_{2}$. Our aim is to reconstruct an image of resolution $M_{1} \times M_{2}$, where $M_{1}=L_{1} \times N_{1}$ and $M_{2}=L_{2} \times N_{2}$. To maintain the aspect ratio of the reconstructed image, we consider the case where $L_{1}=L_{2}=L$ only. For simplicity, we assume that $L$ is an even number in the following discussion.

In order to have enough information to resolve the high-resolution image, there are subpixel displacements between sensors. In the ideal case, the sensors are shifted from each other by a value proportional to $T_{1} / L \times T_{2} / L$. However, in practice there can be small perturbations around these ideal subpixel locations due to imperfections of the mechanical imaging system. Thus, for $l_{1}, l_{2}=0,1, \ldots, L-1$ with $\left(l_{1}, l_{2}\right) \neq(0,0)$, the horizontal and vertical displacements $d_{l_{1} l_{2}}^{x}$ and $d_{l_{1} l_{2}}^{y}$ of the $\left[l_{1}, l_{2}\right]$-th sensor with respect to the $[0,0]$-th reference sensor are given by

$$
d_{l_{1} l_{2}}^{x}=\frac{T_{1}}{L}\left(l_{1}+\epsilon_{l_{1} l_{2}}^{x}\right) \quad \text { and } \quad d_{l_{1} l_{2}}^{y}=\frac{T_{2}}{L}\left(l_{2}+\epsilon_{l_{1} l_{2}}^{y}\right) \text {. }
$$

Here, $\epsilon_{l_{1} l_{2}}^{x}$ and $\epsilon_{l_{1} l_{2}}^{y}$ denote respectively the normalized horizontal and vertical displacement errors.

We remark that the parameters $\epsilon_{l_{1} l_{2}}^{x}$ and $\epsilon_{l_{1} l_{2}}^{y}$ can be obtained by manufacturers during camera calibration. We assume that

$$
\left|\epsilon_{l_{1} l_{2}}^{x}\right|<\frac{1}{2} \quad \text { and } \quad\left|\epsilon_{l_{1} l_{2}}^{y}\right|<\frac{1}{2} .
$$

For if not, the low-resolution images observed from two different sensors will be overlapped so much that the information is not sufficient to reconstruct the high-resolution image (see [3]).

Let $f$ be the original scene. Then, the observed low-resolution image $g_{l_{1} l_{2}}$ from the $\left(l_{1}, l_{2}\right)$ th sensor is modeled by (3) shown at the bottom of the next page for $n_{1}=1, \ldots, N_{1}$ and $n_{2}=$ $1, \ldots, N_{2}$. Here $\eta_{l_{1} l_{2}}$ is the noise corresponding to the $\left(l_{1}, l_{2}\right)$-th sensor. We interlace the low-resolution images to form an $M_{1} \times$ $M_{2}$ image by assigning

$$
g\left[L\left(n_{1}-1\right)+l_{1}, L\left(n_{2}-1\right)+l_{2}\right]=g_{l_{1} l_{2}}\left[n_{1}, n_{2}\right] .
$$


Here, $g$ is an $M_{1} \times M_{2}$ image and is called the observed highresolution image.

The continuous-image model in (3) can be discretized by the rectangular rule and approximated by a discrete image model. Let $\mathbf{g}$ and $\mathbf{f}$ be, respectively, the discretizations of $g$ and $f$ using a column ordering. According to the integration formula in (3), the $\left(n_{1}, n_{2}\right)$-th value of $g$ is the average of the values at the neighborhood $\left[n_{1}-\frac{1}{2}, n_{1}+\frac{1}{2}\right] \times\left[n_{2}-\frac{1}{2}, n_{2}+\frac{1}{2}\right]$ of $f$, implying, therefore, that the corresponding blur matrix is banded. The Neumann boundary condition, imposed on the images, assumes that the scene immediately outside is a reflection of the original scene at the boundary, i.e.

$$
f(i, j)=f(k, l) \quad \text { where } \begin{cases}k=1-i, & i<1 \\ k=2 M_{1}+1-i, & i>M_{1} \\ l=1-j, & j<1 \\ l=2 M_{2}+1-j, & j>M_{2} .\end{cases}
$$

Under the Neumann boundary condition, the blurring matrices are still banded matrices with bandwidth $L+1$, but the entries on the upper left part and the lower right part of the matrices are changed. The resulting matrices, denoted by $\mathbf{H}_{l_{1} l_{2}}^{x}\left(\epsilon_{l_{1}, l_{2}}^{x}\right)$ and $\mathbf{H}_{l_{1} l_{2}}^{y}\left(\epsilon_{l_{1}, l_{2}}^{y}\right)$, have a Toeplitz-plus-Hankel structure

$$
\begin{aligned}
& \mathbf{H}_{l_{1} l_{2}}^{x}\left(\epsilon_{l_{1}, l_{2}}^{x}\right)=\frac{1}{L}\left(\begin{array}{cccccc}
\overbrace{1}^{c / 2} & \cdots & 1 & h_{l_{1} l_{2}}^{x+} & & 0 \\
\vdots & \ddots & \ddots & \ddots & \ddots & \\
1 & \ddots & \ddots & \ddots & \ddots & h_{l_{1} l_{2}}^{x+} \\
h_{l_{1} l_{2}}^{x-} & \ddots & \ddots & \ddots & \ddots & 1 \\
\ddots & \ddots & \ddots & \ddots & \ddots & \vdots \\
0 & & h_{l_{1} l_{2}}^{x-} & \underbrace{1}_{L / 2} & \cdots & 1 \\
& & &
\end{array}\right)
\end{aligned}
$$

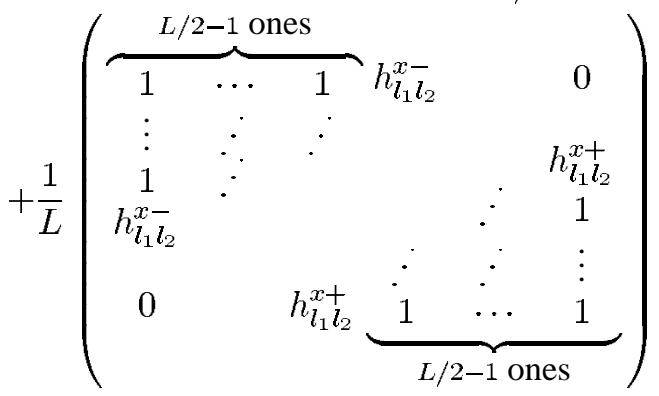

where

$$
h_{l_{1} l_{2}}^{x \pm}=\frac{1}{2} \mp \epsilon_{l_{1} l_{2}}^{x}
$$

and $\mathbf{H}_{l_{1} l_{2}}^{y}\left(\epsilon_{l_{1}, l_{2}}^{y}\right)$ is defined similarly. The blurring matrix corresponding to the $\left(l_{1}, l_{2}\right)$-th sensor under the Neumann boundary condition is given by the Kronecker product

$$
\mathbf{H}_{l_{1} l_{2}}\left(\epsilon_{l_{1}, l_{2}}\right)=\mathbf{H}_{l_{1} l_{2}}^{x}\left(\epsilon_{l_{1}, l_{2}}^{x}\right) \otimes \mathbf{H}_{l_{1} l_{2}}^{y}\left(\epsilon_{l_{1}, l_{2}}^{y}\right) .
$$

The blurring matrix for the whole sensor array is made up of blurring matrices from each sensor

$$
\mathbf{H}_{L}(\epsilon)=\sum_{l_{1}=0}^{L-1} \sum_{l_{2}=0}^{L-1} \mathbf{D}_{l_{1} l_{2}} \mathbf{H}_{l_{1} l_{2}}\left(\epsilon_{l_{1}, l_{2}}\right)
$$

Here, $\mathbf{D}_{l_{1} l_{2}}$ are diagonal matrices with diagonal elements equal to 1 if the corresponding component of $\mathbf{g}$ comes from the $\left(l_{1}, l_{2}\right)$-th sensor and zero otherwise, see [3] for more details. With the Tikhonov regularization, our discretization problem becomes

$$
\left(\mathbf{H}_{L}(\epsilon)^{t} \mathbf{H}_{L}(\epsilon)+\alpha \mathbf{R}\right) \mathbf{f}=\mathbf{H}_{L}(\epsilon)^{t} \mathbf{g}
$$

where $\mathbf{R}$ is the discretization matrix corresponding to the regularization functional $R(f)$ in (2). In this paper, we consider the MAP, $L_{2}$ norm and $H_{1}$ norm regularization functionals. Correspondingly, the matrices $\mathbf{R}$ are the inverse of the covariance matrix of the original image, identity matrix and the discrete Laplacian matrix with the Neumann boundary condition.

\section{ANALYSIS OF DisPLACEMENT ERRORS}

\section{A. Cosine Transform Based Preconditioners}

The linear system (7) will be solved by using the preconditioned conjugate gradient method. Let $\mathbf{C}_{n}$ be the $n \times n$ discrete cosine transform matrix, i.e., the $(i, j)$-th entry of $\mathbf{C}_{n}$ is given by

$$
\sqrt{\frac{2-\delta_{i 1}}{n}} \cos \left(\frac{(i-1)(2 j-1) \pi}{2 n}\right), \quad 1 \leq i, j \leq n
$$

where $\delta_{i j}$ is the Kronecker delta. Note that the matrix-vector product $\mathbf{C}_{n} \mathbf{z}$ can be computed in $O(n \log n)$ operations for any vector $\mathbf{z}$, see ([13], pp. 59-60).

When there are no subpixel displacement errors, i.e., when all $\epsilon_{l_{1}, l_{2}}^{x}=\epsilon_{l_{1}, l_{2}}^{y}=0$, the matrices $\mathbf{H}_{l_{1} l_{2}}^{x}(0)$ and also $\mathbf{H}_{l_{1} l_{2}}^{y}(0)$ are the same for all $l_{1}$ and $l_{2}$. We will denote them simply by $\mathbf{H}_{L}^{x}$ and $\mathbf{H}_{L}^{y}$. We claim that in this case, the blurring matrix $\mathbf{H}_{L} \equiv \mathbf{H}_{L}(0)=\mathbf{H}_{L}^{x} \otimes \mathbf{H}_{L}^{y}$ can always be diagonalized by the discrete cosine transform matrix.

Lemma 1: For any given even integer $L$, we have

$$
\left\|\mathbf{H}_{L}(\epsilon)-\mathbf{H}_{L}\right\|_{2} \leq \frac{4}{L} \epsilon^{*}
$$

where $\epsilon^{*}=\max _{0 \leq l_{1}, l_{2} \leq L-1}\left\{\left|\epsilon_{l_{1} l_{2}}^{x}\right|,\left|\epsilon_{l_{1} l_{2}}^{y}\right|\right\}$.

$$
g_{l_{1} l_{2}}\left[n_{1}, n_{2}\right]=\int_{T_{2}\left(n_{2}-\frac{1}{2}\right)+d_{l_{1} l_{2}}^{y}}^{T_{2}\left(n_{2}+\frac{1}{2}\right)+d_{l_{1} l_{2}}^{y}} \int_{T_{1}\left(n_{1}-\frac{1}{2}\right)+d_{l_{1} l_{2}}^{x}}^{T_{1}\left(n_{1}+\left(\frac{1}{2}\right)+d_{l_{1} l_{2}}^{x}\right.} f\left(x_{1}, x_{2}\right) d x_{1} d x_{2}+\eta_{l_{1} l_{2}}\left[n_{1}, n_{2}\right]
$$


Proof: For $l_{1}, l_{2}=0,1, \ldots, L-1$, we get

$$
\begin{aligned}
& \mathbf{H}_{l_{1} l_{2}}^{x}\left(\epsilon_{l_{1} l_{2}}^{x}\right)-\mathbf{H}_{L}^{x}
\end{aligned}
$$

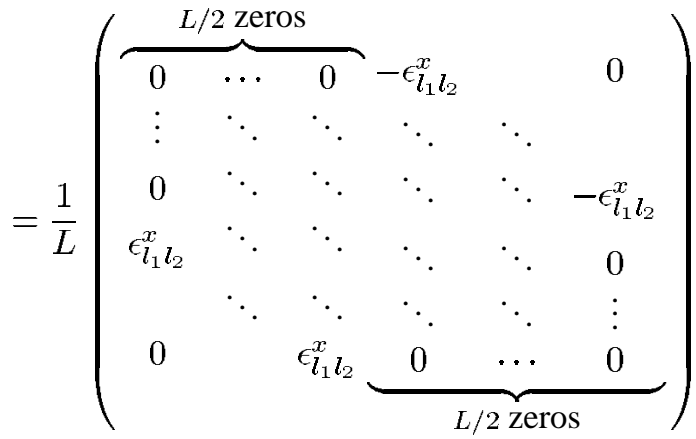

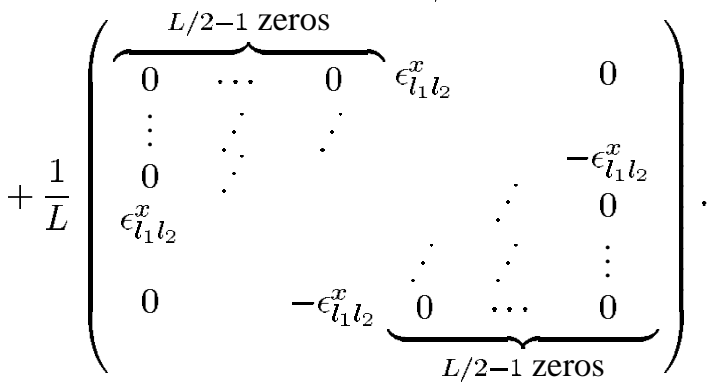

We note that each row or each column of $\mathbf{H}_{l_{1} l_{2}}^{x}\left(\epsilon_{l_{1} l_{2}}^{x}\right)-\mathbf{H}_{L}^{x}$ has at most two nonzero entries. Therefore, we have

$$
\begin{aligned}
\left\|\mathbf{H}_{l_{1} l_{2}}^{x}\left(\epsilon_{l_{1} l_{2}}^{x}\right)-\mathbf{H}_{L}^{x}\right\|_{1} & =\left\|\mathbf{H}_{l_{1} l_{2}}^{x}\left(\epsilon_{l_{1} l_{2}}^{x}\right)-\mathbf{H}_{L}^{x}\right\|_{\infty} \\
& =\frac{2}{L}\left|\epsilon_{l_{1} l_{2}}^{x}\right|
\end{aligned}
$$

It implies that

$$
\begin{aligned}
& \left\|\mathbf{H}_{l_{1} l_{2}}^{x}\left(\epsilon_{l_{l_{l}} l_{2}}^{x}\right)-\mathbf{H}_{L}^{x}\right\|_{2} \\
& \quad \leq \sqrt{\left\|\mathbf{H}_{l_{1} l_{2}}^{x}\left(\epsilon_{l_{1} l_{2}}^{x}\right)-\mathbf{H}_{L}^{x}\right\|_{1}\left\|\mathbf{H}_{l_{1} l_{2}}^{x}\left(\epsilon_{l_{1} l_{2}}^{x}\right)-\mathbf{H}_{L}^{x}\right\|_{\infty}} \\
& \quad=\frac{2}{L}\left|\epsilon_{l_{1} l_{2}}^{x}\right| .
\end{aligned}
$$

By a similar argument, we also obtain $\left\|\mathbf{H}_{l_{1} l_{2}}^{y}\left(\epsilon_{l_{1} l_{2}}^{y}\right)-\mathbf{H}_{L}^{y}\right\|_{2} \leq$ $2\left|\epsilon_{l_{1} l_{2}}^{y}\right| / L$. We note from (5) that each row or each column of $\mathbf{H}_{l_{1} l_{2}}^{x}\left(\epsilon_{l_{1} l_{2}}^{x}\right)$ has $(L-1)$ entries of 1 , one entry of $h_{l_{1} l_{2}}^{x-}$ and one entry of $h_{l_{1} l_{2}}^{x+}$. Therefore

and

$$
\left\|\mathbf{H}_{l_{1} l_{2}}^{x}\left(\epsilon_{l_{1} l_{2}}^{x}\right)\right\|_{1}=\left\|\mathbf{H}_{l_{1} l_{2}}^{x}\left(\epsilon_{l_{1} l_{2}}^{x}\right)\right\|_{\infty}=1
$$

$$
\left\|\mathbf{H}_{L}^{y}\right\|_{1}=\left\|\mathbf{H}_{L}^{y}\right\|_{\infty}=1 .
$$

Hence, both $\left\|\mathbf{H}_{L}^{y}\right\|_{2}$ and $\left\|\mathbf{H}_{l_{1} l_{2}}^{x}\left(\epsilon_{l_{1} l_{2}}^{x}\right)\right\|_{2}$ are less than or equal to 1 . Since $\mathbf{H}_{L}^{y}$ is symmetric and [1...1 $]^{t}$ (or its scalar multiple) is the eigenvector of $\mathbf{H}_{L}^{y}$, the corresponding eigenvalue is equal to 1 . This fact can also be inferred from the expression for the eigenvalues of $\mathbf{H}_{L}^{y}$. To wit, the eigenvalues of the spatially invariant blur matrix $\mathbf{H}_{L}^{y}$ are [15]

$$
\begin{aligned}
\lambda_{i}\left(\mathbf{H}_{L}^{y}\right)=\frac{4}{L} \cos ^{2}\left(\frac{(i-1) \pi}{2 M_{2}}\right) & \\
& \times p_{L}\left(\frac{(i-1) \pi}{M_{2}}\right), \quad 1 \leq i \leq M_{2}
\end{aligned}
$$

where for some positive integer $k$

$$
\begin{aligned}
& p_{L}\left(\frac{(i-1) \pi}{M_{2}}\right) \\
& = \begin{cases}\sum_{j=1}^{L / 4} \cos \left(\frac{(i-1)(2 j-1) \pi}{M_{2}}\right), & L=4 k, \\
\frac{1}{2}+\sum_{j=1}^{(L-2) / 4} \cos \left(\frac{(i-1) 2 j \pi}{M_{2}}\right), & \text { else. }\end{cases}
\end{aligned}
$$

The largest eigenvalue of $H_{L}^{y}$ is equal to 1 , and therefore $\left\|\mathbf{H}_{L}^{y}\right\|_{2}=1$. Using these results, we derive

$$
\begin{aligned}
&\left\|\mathbf{H}_{l_{1} l_{2}}\left(\epsilon_{l_{1} l_{2}}\right)-\mathbf{H}_{L}\right\|_{2} \\
&=\| \mathbf{H}_{l_{1} l_{2}}^{x}\left(\epsilon_{l_{1} l_{2}}^{x}\right) \otimes\left[\mathbf{H}_{l_{1} l_{2}}^{y}\left(\epsilon_{l_{1} l_{2}}^{y}\right)-\mathbf{H}_{L}^{y}\right] \\
& \quad-\left[\mathbf{H}_{L}^{x}-\mathbf{H}_{l_{1} l_{2}}^{x}\left(\epsilon_{l_{1} l_{2}}^{x}\right)\right] \otimes \mathbf{H}_{L}^{y} \|_{2} \\
& \leq\left\|\mathbf{H}_{l_{1} l_{2}}^{x}\left(\epsilon_{l_{1} l_{2}}^{x}\right) \otimes\left[\mathbf{H}_{l_{1} l_{2}}^{y}\left(\epsilon_{l_{1} l_{2}}^{y}\right)-\mathbf{H}_{L}^{y}\right]\right\|_{2} \\
& \quad+\|\left[\mathbf{H}_{L}^{x}-\mathbf{H}_{l_{1} l_{2}}^{x}\left(\epsilon_{l_{1} l_{2}}^{x}\right) \otimes \mathbf{H}_{L}^{y} \|_{2}\right. \\
& \leq\left\|\mathbf{H}_{l_{1} l_{2}}^{x}\left(\epsilon_{l_{1} l_{2}}^{x}\right)\right\|_{2} \cdot\left\|\mathbf{H}_{l_{1} l_{2}}^{y}\left(\epsilon_{l_{1} l_{2}}^{y}\right)-\mathbf{H}_{L}^{y}\right\|_{2} \\
& \quad+\left\|\mathbf{H}_{L}^{x}-\mathbf{H}_{l_{1} l_{2}}^{x}\left(\epsilon_{l_{1} l_{2}}^{x}\right)\right\|_{2} \cdot\left\|\mathbf{H}_{L}^{y}\right\|_{2} \\
&=\left\|\mathbf{H}_{l_{1} l_{2}}^{x}\left(\epsilon_{l_{1} l_{2}}^{x}\right)\right\|_{2} \cdot \frac{2}{L}\left|\epsilon_{l_{1} l_{2}}^{y}\right| \\
& \quad+\frac{2}{L} \mid \epsilon_{l_{1} l_{2}}^{x} \cdot\left\|\mathbf{H}_{L}^{y}\right\|_{2} \\
& \leq \frac{2}{L} \epsilon^{*}+\frac{2}{L} \epsilon^{*}=\frac{4}{L} \epsilon^{*} .
\end{aligned}
$$

Since $\sum_{l_{1}=0}^{L-1} \sum_{l_{2}=0}^{L-1} \mathrm{D}_{l_{1} l_{2}}=\mathbf{I}$, it follows that

$$
\begin{aligned}
\left\|\mathbf{H}_{L}(\epsilon)-\mathbf{H}_{L}\right\|_{2} & =\left\|\sum_{l_{1}=0}^{L-1} \sum_{l_{2}=0}^{L-1} \mathbf{D}_{l_{1} l_{2}} \mathbf{H}_{l_{1} l_{2}}\left(\epsilon_{l_{1}, l_{2}}\right)-\mathbf{H}_{L}\right\|_{2} \\
& =\left\|\sum_{l_{1}=0}^{L-1} \sum_{l_{2}=0}^{L-1} \mathrm{D}_{l_{1} l_{2}}\left[\mathbf{H}_{l_{1} l_{2}}\left(\epsilon_{l_{1}, l_{2}}\right)-\mathbf{H}_{L}\right]\right\|_{2} \\
& \leq\left\|\mathbf{H}_{l_{1} l_{2}}\left(\epsilon_{l_{1}, l_{2}}\right)-\mathbf{H}_{L}\right\|_{2} \leq \frac{4}{L} \epsilon^{*} .
\end{aligned}
$$

When there are subpixel displacement errors, the blurring matrix $\mathbf{H}_{L}(\epsilon)$ has the same banded structure as that of $\mathbf{H}_{L}$, but with some entries slightly perturbed. It is a nonsymmetric block-Toeplitz-Hankel-Toeplitz-Hankel block matrix but it can no longer be diagonalized by the discrete cosine transform matrix. Therefore we solve the corresponding linear system by the preconditioned conjugate gradient method. We will use the cosine transform preconditioner $\mathbf{H}_{L}$ of $\mathbf{H}_{L}(\epsilon)$ as the preconditioner.

\section{B. Convergence Analysis}

In this section, we study the convergence rate of the preconditioned conjugate gradient method for solving the linear systems

$$
\left[\mathbf{H}_{L}(\epsilon)^{t} \mathbf{H}_{L}(\epsilon)+\alpha \mathbf{R}\right] \mathbf{f}=\mathbf{H}_{L}(\epsilon)^{t} \mathbf{g}
$$

where $\alpha$ is a positive constant. We remark that when the MAP, $L_{2}$ norm or $H_{1}$ norm regularization functional is used, the 
TABLE I

NUMBER OF PCG ITERATIONS FOR $L=2$

\begin{tabular}{c|cccc|cccc|cccc}
\hline & \multicolumn{4}{|c|}{$\alpha=0.1$} & \multicolumn{4}{c|}{$\alpha=0.01$} & \multicolumn{4}{c}{$\alpha=0.001$} \\
$M / \epsilon^{*}$ & 0.0375 & 0.075 & 0.15 & 0.3 & 0.0375 & 0.075 & 0.15 & 0.3 & 0.0375 & 0.075 & 0.15 & 0.3 \\
\hline 32 & 3 & 4 & 5 & 7 & 4 & 5 & 7 & 9 & 4 & 6 & 10 & 18 \\
64 & 3 & 4 & 5 & 7 & 4 & 5 & 7 & 9 & 4 & 6 & 10 & 18 \\
128 & 3 & 4 & 5 & 7 & 4 & 5 & 7 & 9 & 4 & 6 & 10 & 17 \\
256 & 3 & 4 & 5 & 7 & 4 & 5 & 7 & 9 & 4 & 6 & 11 & 17 \\
\hline
\end{tabular}

corresponding regularization matrices $\mathbf{R}$ under the Neumann boundary condition can be diagonalized by the discrete cosine transform matrix. Thus if we use the Neumann boundary condition for both the blurring matrix $\mathbf{H}_{L}$ and the regularization matrix $\mathbf{R}$ then the coefficient matrix $\mathbf{H}_{L}^{t} \mathbf{H}_{L}+\alpha \mathbf{R}$ can be diagonalized by the discrete cosine transform matrix and hence its inversion can be done with three two-dimensional fast cosine transforms (one for finding the eigenvalues of the coefficient matrix and two for transforming the right hand side and the solution vector; see [13] for instance). Thus the total cost of solving the system is of $O\left(M_{1} M_{2} \log M_{1} M_{2}\right)$ operations.

Below we show that the convergence rate of the conjugate gradient method when applied to solve the linear system (8) depends linearly on the displacement errors arising from imperfect subpixel locations.

Theorem 1: Let $\epsilon^{*}=\max _{0<l_{1}, l_{2}<L-1}\left\{\left|\epsilon_{l_{1} l_{2}}^{x}\right|,\left|\epsilon_{l_{1} l_{2}}^{y}\right|\right\}$. Then the spectra of the preconditioned matrix $\left[\mathbf{H}_{L}^{t} \mathbf{H}_{L}+\right.$ $\alpha \mathbf{R}]^{-1}\left[\mathbf{H}_{L}(\epsilon)^{t} \mathbf{H}_{L}(\epsilon)+\alpha \mathbf{R}\right]$ belong to the interval $\left[1-8 c \epsilon^{*} / L, 1+8 c \epsilon^{*} / L\right]$, where $c$ is a positive constant independent of $M_{1}$ and $M_{2}$ and $\epsilon^{*}$.

Proof: We note that

$$
\begin{aligned}
& {\left[\mathbf{H}_{L}^{t} \mathbf{H}_{L}+\alpha \mathbf{R}\right]^{-1}\left[\mathbf{H}_{L}(\epsilon)^{t} \mathbf{H}_{L}(\epsilon)+\alpha \mathbf{R}\right]} \\
& \quad=\mathbf{I}+\left[\mathbf{H}_{L}^{t} \mathbf{H}_{L}+\alpha \mathbf{R}\right]^{-1}\left[\mathbf{H}_{L}(\epsilon)^{t} \mathbf{H}_{L}(\epsilon)-\mathbf{H}_{L}^{t} \mathbf{H}_{L}\right]
\end{aligned}
$$

Since

$$
\left\|\mathbf{H}_{L}^{x}\right\|_{2}=\left\|\mathbf{H}_{L}^{y}\right\|_{2}=1
$$

and

$$
\left\|\mathbf{H}_{l_{1} l_{2}}^{x}\left(\epsilon_{l_{1} l_{2}}^{x}\right)\right\|_{2}=\left\|\mathbf{H}_{l_{1} l_{2}}^{y}\left(\epsilon_{l_{1} l_{2}}^{y}\right)\right\|_{2} \leq 1
$$

(see Lemma 1), we have

$$
\left\|\mathbf{H}_{L}\right\|_{2}=1 \quad \text { and } \quad\left\|\mathbf{H}_{L}(\epsilon)^{t}\right\|_{2} \leq 1
$$

By using these results, we obtain

$$
\begin{aligned}
&\left\|\mathbf{H}_{L}(\epsilon)^{t} \mathbf{H}_{L}(\epsilon)-\mathbf{H}_{L}^{t} \mathbf{H}_{L}\right\|_{2} \\
&=\left\|\mathbf{H}_{L}(\epsilon)^{t}\left(\mathbf{H}_{L}(\epsilon)-\mathbf{H}_{L}\right)+\left(\mathbf{H}_{L}(\epsilon)^{t}-\mathbf{H}_{L}^{t}\right) \mathbf{H}_{L}\right\|_{2} \\
& \leq\left\|\mathbf{H}_{L}(\epsilon)^{t}\right\|_{2} \cdot\left\|\mathbf{H}_{L}(\epsilon)-\mathbf{H}_{L}\right\|_{2} \\
& \quad+\left\|\mathbf{H}_{L}(\epsilon)^{t}-\mathbf{H}_{L}^{t}\right\|_{2} \cdot\left\|\mathbf{H}_{L}\right\|_{2} \\
&=\left(\left\|\mathbf{H}_{L}(\epsilon)^{t}\right\|_{2}+\left\|\mathbf{H}_{L}\right\|_{2}\right) \cdot\left\|\mathbf{H}_{L}(\epsilon)-\mathbf{H}_{L}\right\|_{2} \leq \frac{8}{L} \epsilon^{*} .
\end{aligned}
$$
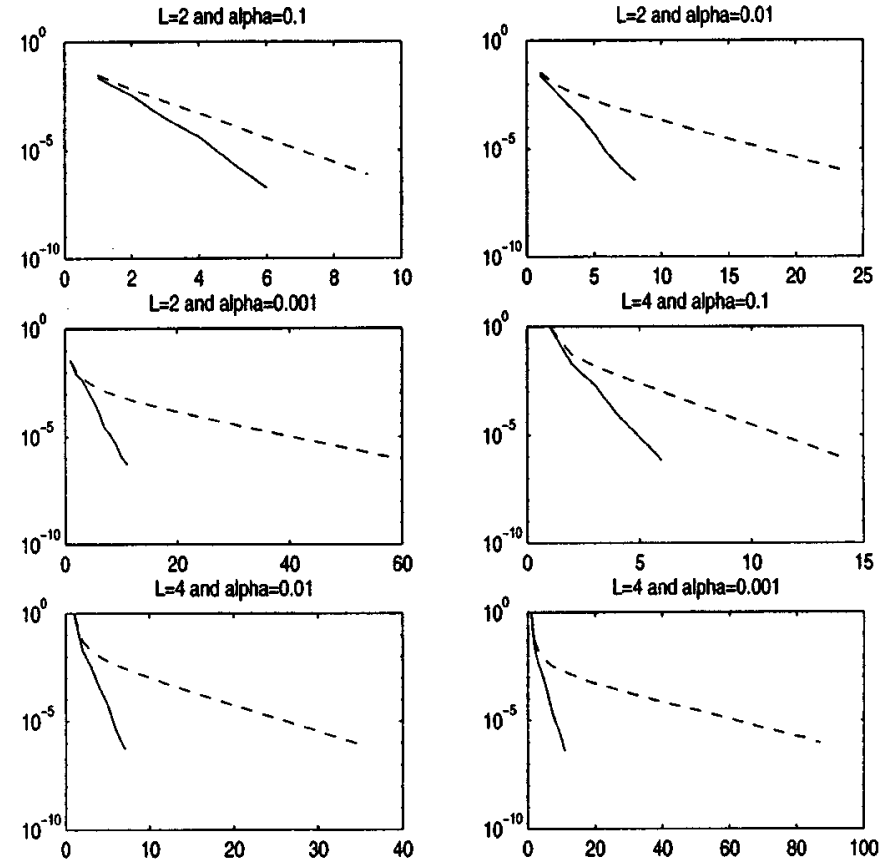

Fig. 1. The convergence curves using preconditioners and without using preconditioners for different $L$ and $\alpha$. The unbroken and broken lines are associated, respectively, with and without the use of preconditioners.

Moreover, it has been shown in [14], [15] that $\|\left(\mathbf{H}_{L}^{t} \mathbf{H}_{L}+\right.$ $\alpha \mathbf{R})^{-1} \|_{2} \leq c$, where $c$ is a positive constant independent of $M_{1}, M_{2}$ and $\epsilon^{*}$. Hence the result follows.

According to the above theorem, we conclude that the spectra of the preconditioned matrices are clustered around 1 for sufficiently small $\epsilon^{*}$. It is of theoretical interest to note that the 2-norm bound obtained above can be improved, though the extra work required to do this is not needed to reach the objective proved next. The reader, interested in understanding better the scopes for further exploiting the algebraic structure inherent in the problem discussed in this paper, might wish to investigate the extent of these improvements. The following theorem proves the convergence rate of the conjugate gradient method when applied to solve the preconditioned systems considered.

Theorem 2: Let $\epsilon^{*}=\max _{0 \leq l_{1}, l_{2} \leq L-1}\left\{\left|\epsilon_{l_{1} l_{2}}^{x}\right|,\left|\epsilon_{l_{1} l_{2}}^{y}\right|\right\}$. If $\epsilon^{*}$ is sufficiently small $\left(\epsilon^{*}<L / 8 c\right)$, then the $k$ th iterate $\mathbf{f}_{k}$ of the conjugate gradient method when applied to solve the linear system (8), satisfies

$$
\frac{\left\|\mathbf{f}_{t}-\mathbf{f}_{k}\right\|_{\mathbf{A}}}{\left\|\mathbf{f}_{t}-\mathrm{f}_{0}\right\|_{\mathbf{A}}} \leq 2\left(\frac{8 c \epsilon^{*}}{L}\right)^{k} .
$$

Here, $\mathbf{f}_{t}$ is the solution of the linear system (8) and $\mathbf{A}=\mathbf{H}_{L}(\epsilon)^{t} \mathbf{H}_{L}(\epsilon)+\alpha \mathbf{R}$. 
TABLE II

NUMBER OF PCG ITERATIONS FOR $L=4$

\begin{tabular}{c|cccc|cccc|cccc}
\hline & \multicolumn{4}{|c|}{$\alpha=0.1$} & \multicolumn{4}{c|}{$\alpha=0.01$} & \multicolumn{5}{c}{$\alpha=0.001$} \\
$M / \epsilon^{*}$ & 0.0375 & 0.075 & 0.15 & 0.3 & 0.0375 & 0.075 & 0.15 & 0.3 & 0.0375 & 0.075 & 0.15 & 0.3 \\
\hline 32 & 3 & 4 & 5 & 7 & 4 & 5 & 7 & 9 & 4 & 6 & 10 & 18 \\
64 & 3 & 4 & 5 & 7 & 4 & 5 & 7 & 9 & 4 & 6 & 10 & 18 \\
128 & 3 & 4 & 5 & 7 & 4 & 5 & 7 & 9 & 4 & 6 & 10 & 17 \\
256 & 3 & 4 & 5 & 7 & 4 & 5 & 7 & 9 & 4 & 6 & 11 & 17 \\
\hline
\end{tabular}

original image

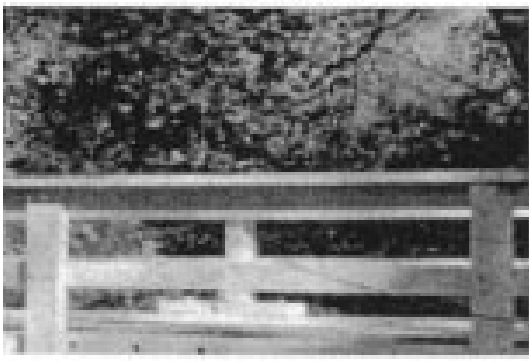

observed high-resolution image

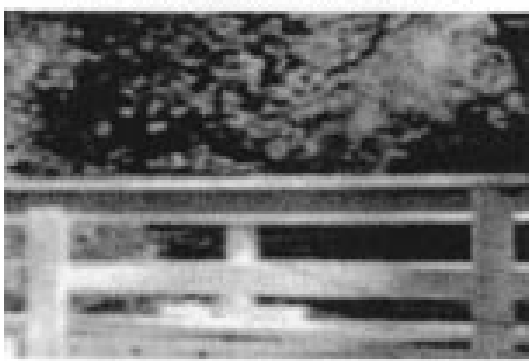

reconstructed image at the 7 iteration

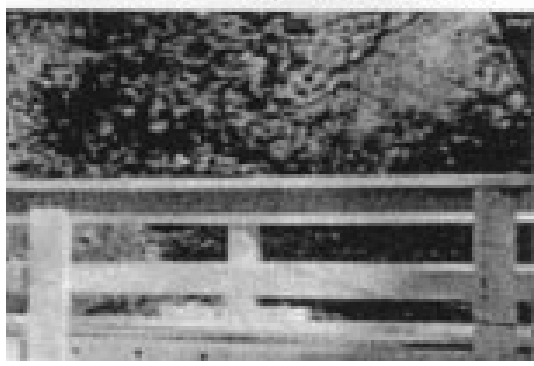

Fig. 2. Reconstructed images when $L=2$ and $\epsilon^{*}=0.15$.

Proof: By using the theorem given in ([1], pp. 569-574), the error vector $\mathbf{f}_{t}-\mathbf{f}_{k}$ at the $k$ th iteration of the conjugate gradient method satisfies

$$
\frac{\left\|\mathbf{f}_{t}-\mathbf{f}_{k}\right\|_{\mathbf{A}}}{\left\|\mathbf{f}_{t}-\mathbf{f}_{0}\right\|_{\mathbf{A}}} \leq 2\left(\frac{b-1}{b+1}\right)^{k}
$$

where

$$
b=\left(\frac{1+8 c \epsilon^{*} / L}{1-8 c \epsilon^{*} / L}\right)^{\frac{1}{2}} \geq 1 .
$$

Since

$$
\frac{b-1}{b+1}=\frac{1-\sqrt{\left[1-\left(8 c \epsilon^{*} / L\right)^{2}\right]}}{\left(8 c \epsilon^{*} / L\right)}<\frac{8 c \epsilon^{*}}{L}<1 .
$$

the result follows. low-resolution image

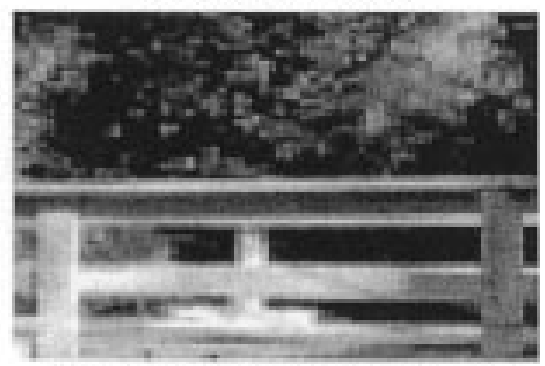

reconstructed image at the 3 iteration

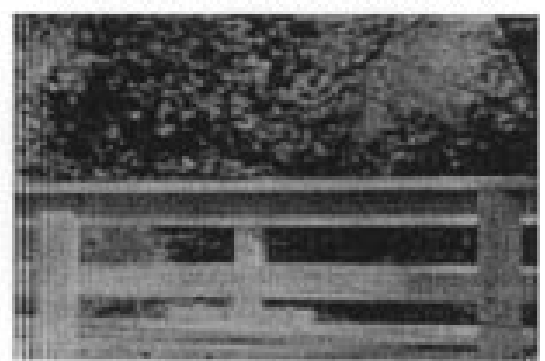

reconstructed image at the 11 iteration

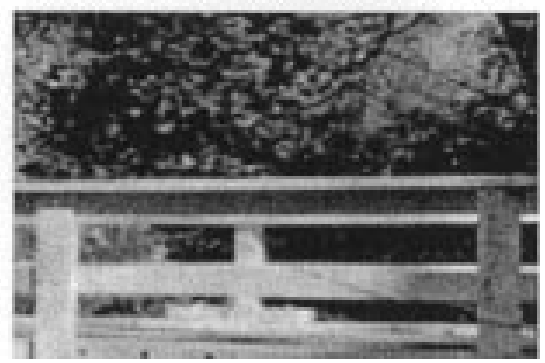

Thus, we conclude that the preconditioned conjugate gradient method applied to (8) with $\alpha>0$ will converge linearly with $\epsilon^{*}$. Since $\mathbf{H}_{L}(\epsilon)$ has only $(L+1)^{2}$ nonzero diagonals, the matrix-vector product $\mathbf{H}_{L}(\epsilon) \mathbf{x}$ can be done in $O\left(L^{2} M_{1} M_{2}\right)$ operations. We note that the preconditioner $\mathbf{H}_{L}^{t} \mathbf{H}_{L}+\alpha \mathbf{R}$ can be diagonalized by the discrete cosine transform matrix. Thus the cost per each iteration is $O\left(M_{1} M_{2} \log M_{1} M_{2}+L^{2} M_{1} M_{2}\right)$ operations. Hence the total cost for finding the high-resolution image vector using the MAP, $L_{2}$-norm or $H_{1}$-norm regularization is $O\left(M_{1} M_{2} \log M_{1} M_{2}+L^{2} M_{1} M_{2}\right)$ operations.

\section{NUMERICAL RESULTS}

In this section, we illustrate the effectiveness of using cosine transform preconditioners for different displacement errors in solving high-resolution image reconstruction problems.

The conjugate gradient method is employed to solve the preconditioned system (8). The stopping criterion is 


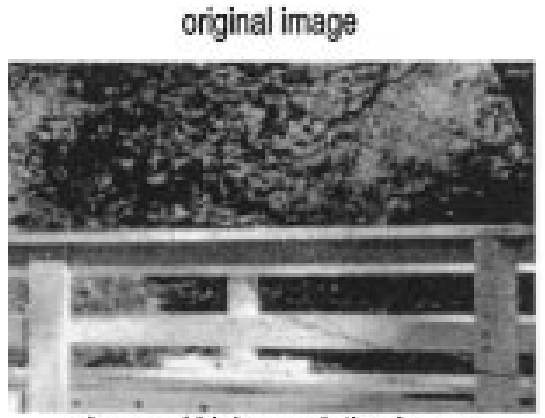

observed high-resolution image

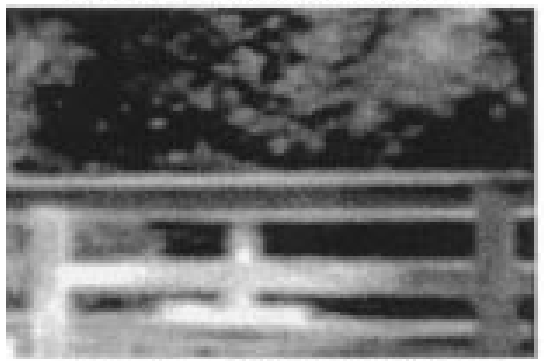

reconstructed image at 7 ileration

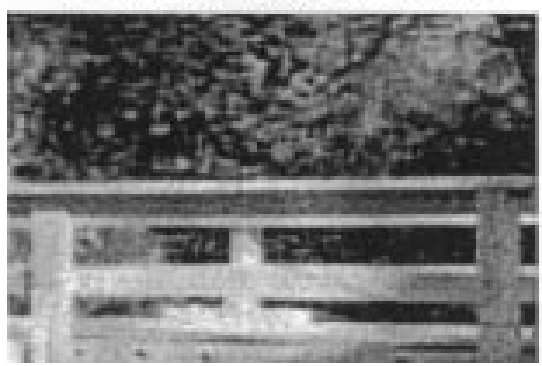

Fig. 3. Reconstructed images when $L=4$ and $\epsilon^{*}=0.15$.

$\left\|\mathbf{r}^{(j)}\right\|_{2} /\left\|\mathbf{r}^{(0)}\right\|_{2}<10^{-6}$, where $\mathbf{r}^{(j)}$ is the normal equations residual after $j$ iterations. In the tests, Gaussian white noise with signal-to-noise ratio of $40 \mathrm{~dB}$ is added to the low-resolution images. Tables I-II show the numbers of PCG iterations, using cosine transform based preconditioners, that are required for convergence for $L=2$ and $L=4$. In the tests, $M_{1}=M_{2}=M$ and the signal-to-noise ratio is $40 \mathrm{~dB}$. We see from the tables that the convergence rate is independent of $M$ for fixed $\alpha$. Moreover, the cosine preconditioned system converges at most linearly with the displacement error $\epsilon^{*}$ as predicted as in Theorem 2. In Fig. 1, we also show the convergence curves (the $y$-axis involves $\left\|\mathrm{r}^{(j)}\right\|_{2} /\left\|\mathrm{r}^{(0)}\right\|_{2}$ and the $x$-axis involves the iteration number) of the cosine transform preconditioned system and the nonpreconditioned system for $L=2$ and 4 . It is clear that when $\alpha$ is small, the convergence of the cosine transform preconditioned system is rapid. In Figs. 2 and 3, we show the 128-by-128 reconstructed images using different PCG iterations when $2 \times 2$ and $4 \times 4$ sensor arrays are used. We find that the reconstructed images already look very good even if the stopping criterion is not reached.

\section{CONCLUSION}

The problem of high-resolution reconstruction from a set of low-resolution images, in general, involves the solving of

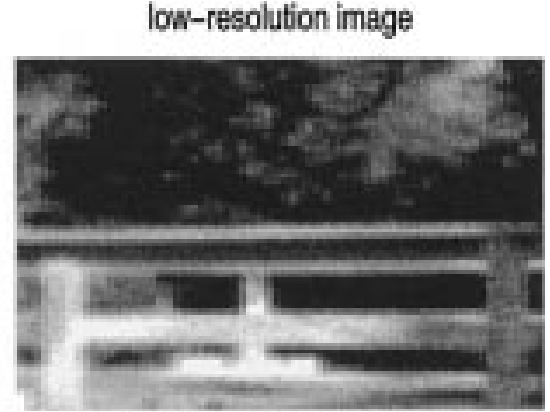

reconstructed image at 3 iteration

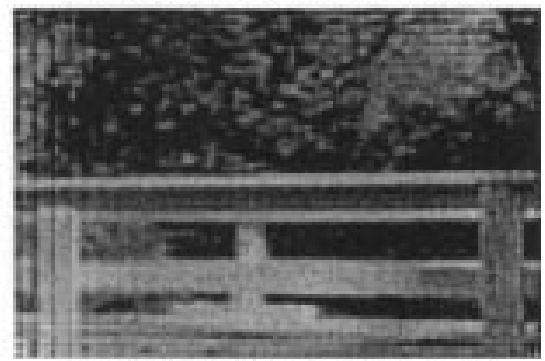

reconstructed image at 10 iteration

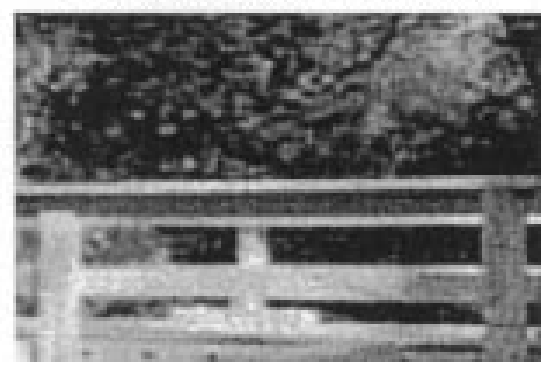

an ill-conditioned and underdetermined large-scale system of linear equations. In spite of the flurry of research in iterative techniques for solution, the computational complexity analysis and numerical issues, though crucial in implementation, have not been adequately addressed. Tikhonov regularization, where the regularization parameter is calculated by the generalized cross-validation technique for the underdetermined system of linear equations, alongwith circulant-type preconditioners have very recently been used for attaining superresolution and computational tractability has been observed from simulations [16].

In this paper, an algorithm for the attainment of high-resolution from low-resolution data acquired by a prefabricated array of sensors, discussed in [3], is analyzed. Reconstruction of a highresolution image from multiple, undersampled, shifted degraded image frames with the inevitable subpixel displacement errors, occuring during the fabrication of charge-coupled device sensor array of sensors, each with a planar subarray of sensing elements used for capturing the image frames, has been a topic of very great research interest. Zero boundary conditions, periodic boundary conditions, and Neumann boundary conditions have been separately imposed on the two-dimensional system model for the reconstruction process. This model is linear shiftvariant and is ill-conditioned requiring the deployment of a regularization technique on the structured system of equations. In the case of Neumann boundary conditions, discrete cosine 
transform based preconditioners have been effective in the high-resolution reconstruction problem [14], [15]. The main contribution of this paper is an analysis and proof of convergence of the iterative method deployed to solve the transform based preconditioned system. The proof of linear convergence of the conjugate gradient method on the displacement errors caused from the imperfect locations of subpixels in the sensor array fabrication process has been given and substantiated by results from simulation. The intriguing possibility of combining this encouraging result with the calculation of the optimum regularization parameter by the $L$-curve [2] gives an analytic foundation for the high-resolution reconstruction process on the image acquisition framework of the CCD sensor array. In the future, attention could be directed toward another model for signal capture that involves a single sensor array with arbitrary shifts between multiple captures, where errors due to motion estimation are ubiquitous.

\section{ACKNOWLEDGMENT}

The authors wish to thank J. Koo, a graduate student assistant in the Department of Electrical Engineering at The Pennsylvania State University, University Park, who proofread this paper by. N. K. Bose would like to thank Dr. S. Basu for his invitation to contribute to this Special Issue.

\section{REFERENCES}

[1] O. Axelsson, Iterative Solution Methods. New York: Cambridge Univ. Press, 1994.

[2] N. K. Bose, S. Lertrattanapanich, and J. Koo, "Advances in superresolution using the $L$-curve," in Proc. Int. Symp. Circuits and Systems, vol. II, Sydney, NSW, Australia, May 2001, pp. 433-436.

[3] N. K. Bose and K. Boo, "High-resolution image reconstruction with multisensors," Int. J. Imaging Syst. Technol., vol. 9, pp. 294-304, 1998

[4] R. Chan, T. Chan, M. Ng, W. Tang, and C. Wong, "Preconditioned iterative methods for high-resolution image reconstruction with multisensors," in Proc. SPIE Symp. Advanced Signal Processing: Algorithms, Architectures, and Implementations, vol. 3461, F. Luk, Ed., San Diego, CA, July 1998, pp. 348-357.

[5] M. Elad and A. Feuer, "Restoration of single superresolution image from several blurred, noisy, and undersampled measured images," IEEE Trans. Image Processing, vol. 6, pp. 1646-1658, 1997.

[6] J. C. Gillete, T. M. Stadtmiller, and R. C. Hardie, "Aliasing reduction in staring infrared imagers using subpixel techniques," Opt. Eng., vol. 34 no. 11, pp. 3130-3137, Nov. 1995 .

[7] R. C. Hardie, K. J. Barnard, J. C. Bognar, E. E. Armstrong, and E. A Watson, "High-resolution image reconstruction from a sequence of rotated and translated frames and its application to an infrared imaging system," Opt. Eng., vol. 37, no. 1, pp. 247-260, Jan. 1998.

[8] G. Jacquemod, C. Odet, and R. Goutte, "Image resolution enhancement using subpixel camera displacement," Signal Processing, vol. 26, pp. 139-146, 1992.

[9] S. P. Kim, N. K. Bose, and H. M. Valenzuela, "Recursive reconstruction of high resolution image from noisy undersampled multiframes," IEEE Trans. Acoust., Speech, Signal Proc., vol. 38, pp. 1013-1027, 1990

[10] S. P. Kim and N. K. Bose, "Reconstruction of 2-D bandlimited discrete signals from nonuniform samples,", in Proc. Inst. Elect. Eng., vol. 137, 1990, pp. 197-203.

[11] S. P. Kim and W. Su, "Recursive high-resolution reconstruction of blurred multiframe images," IEEE Trans. Image Processing, vol. 2, pp. 534-539, 1993

[12] T. Komatsu, K. Aizawa, T. Igarashi, and T. Saito, "Signal-processing based method for acquiring very high resolution images with multiple cameras and its theoretical analysis," in Proc. Inst. Elect. Eng., vol. 140, 1993, pp. 19-25.

[13] M. Ng, R. Chan, and W. Tang, "A fast algorithm for deblurring models with Neumann boundary conditions," SIAM J. Sci. Comput., vol. 21, pp. 851-866, 1999
[14] M. Ng, R. Chan, T. Chan, and A. Yip, "Cosine transform preconditioners for high resolution image reconstruction," Linear Algebra and Applics. vol. 316, pp. 89-104, 2000

[15] M. Ng and A. Yip, "A fast MAP algorithm for high-resolution image reconstruction with multisensors," Multidimensional Syst. Signal Processing, vol. 12, no. 2, pp. 143-164, 2001.

[16] N. Nguyen, P. Milanfar, and G. Golub, "A computationally efficient superresolution image reconstruction algorithm," IEEE Trans. Image Processing, vol. 10, pp. 573-583, 2001.

[17] S. Rhee and M. G. Kang, "Discrete cosine transform based regularized high-resolution image reconstruction algorithm," Opt. Eng., vol. 38, no. 8, pp. 1348-1356, Aug. 1999

[18] R. Schultz and R. Stevenson, "Extraction of high-resolution frames from video sequences," IEEE Trans. Image Processing, vol. 5, pp. 996-1011, 1996.

[19] H. Stark and P. Oskoui, "High-resolution image recovery from imageplane arrays using convex projections," J. Opt. Soc. Amer. A, vol. 6, pp. 1715-1726, 1989

[20] A. Tekalp, M. Ozkan, and M. Sezan, "Superresolution video reconstruction with arbitrary sampling lattices and nonzero aperture time," IEEE Trans. Image Processing, vol. 6, pp. 1064-1076, 1997.

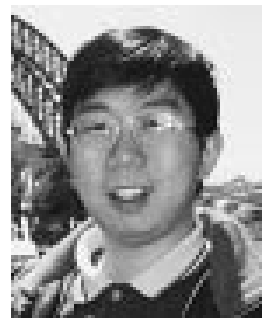

Michael K. Ng was born in Hong Kong, China, in 1967. He received B.Sc. and M.Phil. degrees in mathematics from the University of Hong Kong, Hong Kong, in 1990 and 1993, respectively, and the Ph.D. degree in mathematics from the Chinese University of Hong Kong, Hong Kong, in 1995.

From 1995 to 1997 he was a Research Fellow at the Australian National University, Canberra, Australia. $\mathrm{He}$ is currently an Assistant Professor in the Department of Mathematics at the University of Hong Kong. His research interests are in the areas of data mining, operations research and scientific computing.

Dr. Ng has been selected as one of the recipients of the Outstanding Young Researcher Award of the University of Hong Kong in 2001.

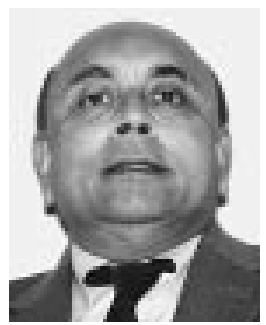

N. K. Bose (S'66-M'67-SM'74-F'81) received the B.Tech. (Hons.), M.S., and Ph.D. degrees in electrical engineering from the Indian Institute of Technology (I.I.T.), Kharagpur, India, Cornell University, Ithaca, NY, and Syracuse University, Syracuse, NY, respectively.

From 1967 to 1986, he was at the University of Pittsburgh, Pittsburgh, PA where he became a Professor of Electrical Engineering and Professor of Mathematics. Currently, he is the HRB-Systems and Fetter Endowment Professor of Electrical Engineering at The Pennsylvania State University, University Park, which he joined in 1986 to become the Singer Professor and Founding Director of the Center for Spatial and Temporal Signal Processing. He was a visiting faculty at several Institutions including the American University of Beirut, Lebanon, the University of Maryland at College Park, CNRS-IRIA and LAAS (Paris, Toulouse), Ruhr Universitaet, Germany, the University of California at Berkeley, and Princeton University. He is the author of Applied Multidimensional Systems Theory (New York: Van Nostrand Reinhold, 1982), Digital Filters (Amsterdam, The Netherlands: Elsevier, 1985; Malabar, FL: Krieger, 1993), the main author as well as Editor of Multidimensional Systems; Progress, Directions, and Open Problems (Amsterdam, the Netherlands: Reidel, 1985), and coauthor of Neural Network Fundamentals with Graphs, Algorithms, and Applications (New York: McGraw-Hill, 1996). He is the founding Editor-in-Chief of the International Journal on Multidimensional Systems and Signal Processing and serves on the editorial boards of several other journals.

Dr. Bose was invited to serve as the plenary keynote speaker on several occasions including, most recently, at the First and Second International Workshops on Multidimensional Systems at Poland in 1998 and 2000, respectively. In 2001, he was an invited plenary speaker in the opening session of the International Conference in Sampling Theory and Its Applications. He received several awards and honors including, most recently, the Fetter Endowment Award (2001-2004), the Alexander Von Humboldt Research Award from Germany in 2000, The Invitational Fellowship from the Japan Society for Promotion of Science in 1999, and an invitation from the United Nations to serve as a technical advisor to the Government of India during 1994-1995 